\title{
Mathematical Relevance of Polynions
}

\author{
S. K. PANDEY
}

\begin{abstract}
In this note we introduce the notion of polynions and discuss their mathematical relevance. We note that the well-known mathematical structures like quaternions, octonions and sedenions etc. are special cases of polynions.
\end{abstract}

Keywords: polynion, quaternion, octonion, sedenions, ring, algebra.

MSC2010: 16D25, 16N40.

\section{Introduction}

The well known quaternions [1-3] were discovered by William Rowan Hamilton. These are very useful in mathematics as well as in physics. Octonions [3-4] were discovered later on the basis of the theory of quaternions. Similarly sedenions [5] are an extention of octonions.

We introduce the notion of polynions, plus and minus polynions. It is noted that the well-known mathematical structures like quaternions, octonions, sedenions are special cases of minus polynions.

The mathematical structures given by minus polynions and plus polynions are quite distinct and have different algebraic properties. It is well-known that Quaternions form a four dimensional non-commutative but associative algebra however the algebra of octonions is neither associative nor commutative. But in the case of the mathematical structures given by plus polynions we retain the both commutativity as well as associativity for multiplication.

In general the notion of plus polynions leads to construct a unital commutative as well as associative algebra of dimension $n+1$ for each $n$ where $n=2^{t}-1 ; t \in N \bigcup\{0\}$. Here $N$ denotes the set of natural numbers. 
In the next section we introduce the notion of polynions. In section 3 we discuss the mathematical relevance of polynions. In the last section we provide conclusions.

\section{Polynions}

Definition 2.1: Polynion: Let $p$ be an expression of the form $p=a_{0}+a_{1} e_{1}+a_{2} e_{2}+\ldots+a_{n} e_{n}$. If $e_{1}^{2}=e_{2}^{2}=\ldots=e_{n}^{2}=-1 \quad$ or $\quad e_{1}^{2}=e_{2}^{2}=\ldots=e_{n}^{2}=1$ then $p$ is called a polynion. Here $a_{0}, a_{1}, a_{2}, \ldots, a_{n}$ are real numbers and $n=2^{t}-1 ; t \in N \bigcup\{0\}$. Here $N$ denotes the set of natural numbers.

Definition 2.2: Minus Polynion: If $e_{1}^{2}=e_{2}^{2}=\ldots=e_{n}^{2}=-1$ and $e_{i} e_{j}+e_{j} e_{i}=0$ for each $e_{i}, e_{j}$ such that $e_{i} \neq e_{j}, \quad 1 \leq i \leq n, 1 \leq j \leq n$ then $p=a_{0}+a_{1} e_{1}+a_{2} e_{2}+\ldots+a_{n} e_{n}$ is called the minus polynion. Let $U_{n}^{-}=\left\{ \pm 1, \pm e_{1}, \pm e_{2}, \ldots, \pm e_{n}\right\}$. Then the set $U_{n}^{-}$of all unit minus polynions forms a group under the multiplication of unit minus polynions for each $n \leq 3$. If $n \geq 7$ then $U_{n}^{-}$satisfies each condition to be a group except associativity.

Definition 2.2: Plus Polynion: If $e_{1}^{2}=e_{2}^{2}=\ldots=e_{n}^{2}=1$ and $e_{i} e_{j}+e_{j} e_{i}=2 e_{i} e_{j}$ for each $e_{i}, e_{j}$ such that $e_{i} \neq e_{j} ; 1 \leq i \leq n, \quad 1 \leq j \leq n$ then $p=a_{0}+a_{1} e_{1}+a_{2} e_{2}+\ldots+a_{n} e_{n}$ is called the plus polynion. Let $U_{n}^{+}=\left\{1, e_{1}, e_{2}, \ldots, e_{n}\right\}$. Then the set $U_{n}^{+}$of all unit plus polynions forms a commutative group of order $2^{t}$ under the multiplication of unit plus polynions. In this group each element is self inverse.

It shuld be emphasized that multiplication of unit plus polynions is carried out in the same way as it is done in the case of unit minus polynions but we replace minus sign by plus sign in the case of unit plus polynions. By this we retain commutativity and associtaivity of multiplication for unit plus polynions. We illustrate this by means of the following example.

Let $n=3$ then in the case of unit minus polynions we have (for $e_{i} \neq e_{j}$ ) $e_{1} e_{2}=-e_{2} e_{1}=e_{3}$, $e_{2} e_{3}=-e_{3} e_{2}=e_{1}$ and $e_{3} e_{1}=-e_{1} e_{3}=e_{2}$. However in the case of unit plus polynions this becomes $e_{1} e_{2}=e_{2} e_{1}=e_{3}, e_{2} e_{3}=e_{3} e_{2}=e_{1}$ and $e_{3} e_{1}=e_{1} e_{3}=e_{2}$.

In the next section we discuss about the sets of all minus polynions and plus polynions and provide multiplication rule for $e_{i}$ and $e_{j}$ in the case of plus polynions for $n=1,3$ and 7 . 


\section{Mathematical Relevance of Polynoins}

We consider the case of minus polynions and plus polynions separately.

Case I: Minus Polynions: Let $P_{n}^{-}$denotes the set of all minus polynion. Let us take $n=0$ then it is easy to see that the set $P_{n}^{-}$of all minus polynions reduces to the set of real numbers. If we take $n=1$ then $P_{n}^{-}$reduces to the set of complex numbers. Similarly one may see that $P_{n}^{-}$ reduces to the set of all quaternions and octonions for $n=3$ and 7 respectively. Further if we take $n=15$ then $P_{n}^{-}$gives the set of all sedenions.

It may be noted that real numbers, complex numbers, quaternions, octonions and sedenions are well-known mathematical structures and so the multiplication rule for them can be found in mathematical literatures. Therefore we do not discuss the multiplication rule for $e_{i}$ and $e_{j}$ in these cases. For further details on quaternions, octonions and sedenions one may refer [3-5].

We now come to the set of plus polynions, say $P_{n}^{+}$, and describe the multiplication rule for $e_{i}$ and $e_{j}$ in this case.

Case II: Plus Polynions: We see that the set $P_{n}^{-}$of all minus polynions contains the set $R$ of real numbers as well as the set $C$ of complex numbers. However the set $P_{n}^{+}$of all plus polynions contains the set $R$ of real numbers but not the set $C$ of complex numbers. Below we discuss about the set $P_{n}^{+}$of plus polynions for $n=1,3$ and 7 ( for $n=0, P_{n}^{+}$reduces to the set of real numbers itself).

If we take $n=1$ then we can write $P_{1}^{+}=\left\{a+e_{1} b: a, b \in R\right\}$. Clearly $P_{1}^{+}$gives a special class of numbers which is neither real nor complex but it looks like complex numbers.

One may easily verify that $P_{1}^{+}$is a commutative ring with identity under addition and multiplication of polynions defined below.

Let $p_{1}=a+e_{1} b \in R_{1}$ and $p_{2}=c+e_{1} d \in R_{1}$ then the sum of $p_{1}$ and $p_{2}$ is defined by $p_{1}+p_{2}=$ $\left(a+e_{1} b\right)+\left(c+e_{1} d\right)=(a+c)+e_{1}(b+d)$ and the product of $p_{1}$ and $p_{2}$ is defined by $p_{1} p_{2}=\left(a+e_{1} b\right)\left(c+e_{1} d\right)=(a c+b d)+e_{1}(b c+a d)$.

Moreover one may see that $P_{1}^{+}$is a unital commutative and associative real algebra of dimension two but unlike the algebra of complex numbers this algebra has zero divisors. 
If we take $n=3$ then the set of all plus polynions reduces to $P_{3}^{+}=\left\{a+e_{1} b+e_{2} c+e_{3} d: a, b, c, d \in R\right\}$. Clearly $P_{3}^{+}$gives a set which looks like the set of all quaternions. As already explained in this case we have $e_{1}^{2}=e_{2}^{2}=e_{3}^{2}=1$. Also, $e_{1} e_{2}=e_{2} e_{1}=e_{3}$, $e_{2} e_{3}=e_{3} e_{2}=e_{1}$ and $e_{3} e_{1}=e_{1} e_{3}=e_{2}$. It may be noted that unlike usual Hamilton quaternions $P_{3}^{+}$forms a unital commutative algebra of dimension four. We have retained commutativity as it is clear from the defining relations of $e_{i}$ and $e_{j}$. It may be verified that this algebra has zero divisors.

One may easily verify that in this case the set $U_{3}^{+}=\left\{1, e_{1}, e_{2}, e_{3}\right\}$ of unit plus polynions forms the Klein's group under multiplication.

If we take $n=7$ then the set of all plus polynions reduces to $P_{7}^{+}=\left\{a_{0}+a_{1} e_{1}+a_{2} e_{2}+a_{3} e_{3}+a_{4} e_{4}+a_{5} e_{5}+a_{6} e_{6}+a_{7} e_{7}: a_{i} \in R\right\}$. Clearly $P_{7}^{+}$gives a set which looks like the set of all octonions. As already explained in this case we have $e_{1}^{2}=e_{2}^{2}=e_{3}^{2}=\ldots=e_{7}^{2}=1$. The multiplication of $e_{i}$ and $e_{j}$ such that $e_{i} \neq e_{j} ; 1 \leq i \leq 7$, $1 \leq j \leq 7$ can be given in several ways. For example we may define that

$$
\begin{array}{llll}
e_{1} e_{2}=e_{2} e_{1}=e_{4}, & e_{1} e_{3}=e_{3} e_{1}=e_{6} ; & e_{1} e_{4}=e_{4} e_{1}=e_{2}, \quad e_{1} e_{5}=e_{5} e_{1}=e_{7}, \quad e_{1} e_{6}=e_{6} e_{1}=e_{3}, \\
e_{1} e_{7}=e_{7} e_{1}=e_{5} ; & e_{2} e_{3}=e_{3} e_{2}=e_{5}, & e_{2} e_{4}=e_{4} e_{2}=e_{1}, \quad e_{2} e_{5}=e_{5} e_{2}=e_{3}, \quad e_{2} e_{6}=e_{6} e_{2}=e_{7}, \\
e_{2} e_{7}=e_{7} e_{2}=e_{6} ; & e_{3} e_{4}=e_{4} e_{3}=e_{7}, & e_{3} e_{5}=e_{5} e_{3}=e_{2}, \quad e_{3} e_{6}=e_{6} e_{3}=e_{1}, \quad e_{3} e_{7}=e_{7} e_{3}=e_{4} ; \\
e_{4} e_{5}=e_{5} e_{4}=e_{6}, & e_{4} e_{6}=e_{6} e_{4}=e_{5}, \quad e_{4} e_{7}=e_{7} e_{4}=e_{3} ; \quad e_{5} e_{6}=e_{6} e_{5}=e_{4}, \quad e_{5} e_{7}=e_{7} e_{5}=e_{1} ; \\
e_{6} e_{7}=e_{7} e_{6}=e_{2} . &
\end{array}
$$

It may be noted that unlike usual octonions $P_{7}^{+}$forms a unital commutative as well as associative algebra of dimension eight. We have retained commutativity and associativity as it is clear from the defining relations of unit plus octonions. It may be verified that this algebra has zero divisors.

One may easily verify that in this case the set $U_{7}^{+}=\left\{1, e_{1}, e_{2}, e_{3}, e_{4}, e_{5}, e_{6}, e_{7}\right\}$ of unit plus polynions forms a multiplicative group of order eight in which each element is self inverse.

Similarly it may be seen that for $n=15$, the set $P_{n}^{+}$of all plus polynions gives a mathematical structure corresponding to the sedenions.

\section{Conclusions.}


It is seen that quaternions [1-3], octonions [3-4] and sedenions [5] are special cases of minus polynions. The notion of plus polynions leads to define new mathematical structures corresponding to each structure given by the minus polynions. Moreover in the case of plus polynions we retain commutativity and associativity under multiplication and thus the algebra of plus polynions $P_{n}^{+}$is commutative as well as associative for each $n=2^{t}-1 ; t \in N \bigcup\{0\}$ and these algebras have zero divisors whenever $n>0$. One may consider these notions for further possible research.

\title{
References
}

[1] T. Y. Lam, A First Course in Noncommutative Rings, Springer, 2001.

[2] M. Artin, Algebra, Prentice-Hall of India, Pvt. Ltd., New Delhi, 2000.

[3] J. H. Conway, D. A. Smith, On Quaternions and Octonions: Their Geometry, Arithmetic and Symmetry, CRC Press, 2003.

[4] J. C. Baez, The Octonions, Bulletin of the American Mathematical Society, New Series, 39 (2), 145-205, 2002.

[5] K. Imaeda, M. Imaeda, Sedenions: algebra and analysis, Applied Mathematics and Computation, 115 (2), 77-88, 2000.

\author{
S. K. PANDEY, \\ Department of Mathematics, SPUP, Jodhpur, India. \\ E-mail: skpandey12@gmail.com
}

